\title{
INHERITED VENOUS BEADING
}

\author{
BERTRAND PIGUET, MIRJAM GROSS-JENDROSKA, FRANK G. HOLZ and ALAN C. BIRD \\ London
}

\begin{abstract}
SUMMARY
Inherited retinal venous beading is a rare autosomal disorder. We describe three affected members in three generations of a single family. It is evident that there may be gross asymmetry of affection between the two eyes, and highly variable expressivity such that the diagnosis may not be evident without a family survey. One patient had remarkable spontaneous recovery of vision.
\end{abstract}

Recently, two American pedigrees have been described with an autosomal dominant condition which was named 'inherited venous beading'. The disorder is characterised by prominent, irregular, segmental beading of the retinal veins, with or without conjunctival vascular abnormálities. ${ }^{1.2}$ Some patients developed retinopathy consisting of microaneurysms, altered vascular permeability with exudation, retinal haemorrhages or even areas of retinal infarction.

Our report draws attention to this unusual mendelian condition, by recording the first European family known to be affected. Despite extensive ocular and systemic evaluation, the pathogenesis of this condition still remains obscure.

\section{PATIENTS}

The proband (case III-5) was referred because of recent visual loss in his right eye. His father (case II-2) was similarly affected and had also lost vision in one eye. The grandmother was mildly affected and had not suffered visual loss (Fig. 1).

\section{Patient III-5}

The proband presented initially with symptoms of floaters in the left eye at the age of 17 years. His visual acuity was $6 / 4$ with the right eye and 6/6 with the left. There was a large intraretinal haemorrhage above the left disc which had broken into the vitreous cavity. Streaks of hard white exudates were present radiating from the fovea and extending to the disc margin. The retinal veins in both eyes

From: Department of Clinical Ophthalmology, Moorfields Eye Hospital and the Institute of Ophthalmology, London, UK.

Correspondence to: Professor Alan C. Bird, FRCS, Professorial Unit, Moorfields Eye Hospital, City Road, London EC1V 2PD, UK. Fax: 071-2519350. were described as enlarged with marked beading. Fluorescein angiography showed no dye leakage in either eye and no capillary closure was present. The haemorrhage subsequently resolved and the vision returned to normal.

Nine years later, the patient had sudden blurring of vision with the right eye. At this time, the visual acuity was $6 / 60$ with the right eye and $6 / 5$ with the left. The conjunctival veins were normal, and there were no opacities in the cornea or the lens. Intraocular pressure was $16 \mathrm{mmHg}$ in both eyes. There was marked beading of the first- and second-order retinal veins in the right eye which extended to the equator. A 3-disc diameter area of superficial and intraretinal haemorrhage was seen temporal to the disc involving the fovea with serous detachment of the retina and intraretinal exudates (Fig. 2a). In the left fundus there were a few small hard exudates temporally to the fovea, pigmentary changes centrally, prominent beading of the retinal veins and microvascular changes in the inferior half of the posterior pole (Fig. 2b,c).

Three months later the vision had improved to $6 / 36$ with the right eye, and the retinal haemorrhages and exudates had partially cleared. Marked cystoid macular oedema was present, associated with some secondary pigment hyperplasia in the fovea (Fig. 3a). Fluorescein angiography of the right eye showed intraretinal microvascular changes, mainly in the form of telangiectases in the territory of the superior temporal retinal vein (Fig. 3b).

Over the following 9 months, right eye visual acuity improved to $6 / 9$, and apart from venous beading the vascular changes resolved (Fig. 3c).

The past medical history of this healthy patient was unremarkable. At the time of the second ocular episode, the red blood cell count, the platelet count and the blood film were normal. The haemoglobin was $14.1 \mathrm{~g} / \mathrm{dl}$ with a normal electrophoresis. The white blood cell count was normal. Quantitative cell counts were as follows: polymorph neutrophils, $2850 / \mathrm{mm}^{3}$; lymphocytes, $2050 / \mathrm{mm}^{3}$; monocytes, $50 / \mathrm{mm}^{3}$; eosinophils $50 / \mathrm{mm}^{3}$. The results of the following tests were normal: prothrombin index, clot retraction, serum protein electrophoresis, serum immunoglobulin, cryoglobulin levels, electrolytes, cholesterol, triglycerides and fasting blood sugar, renal and hepatic function. Osmotic fragility was not tested given the

Eye (1994) 8, 84-88 C 1994 Royal College of Ophthalmologists 


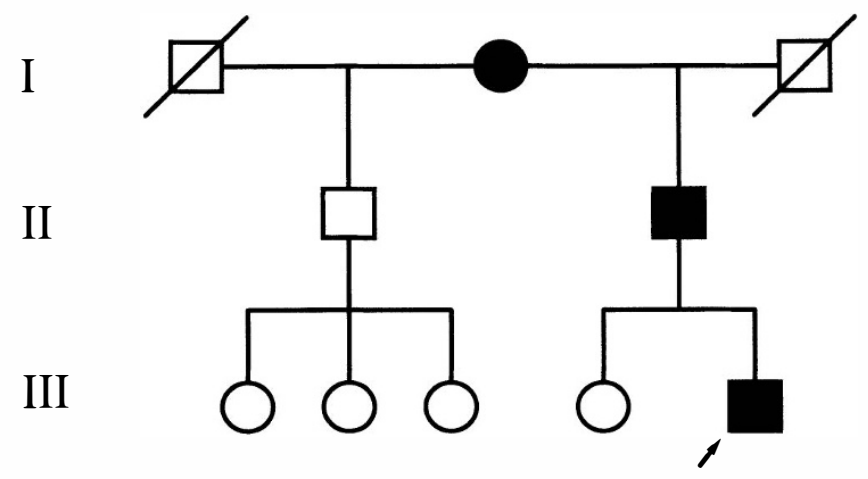

Fig. 1. Pedigree of family. The proband is indicated by an arrow.

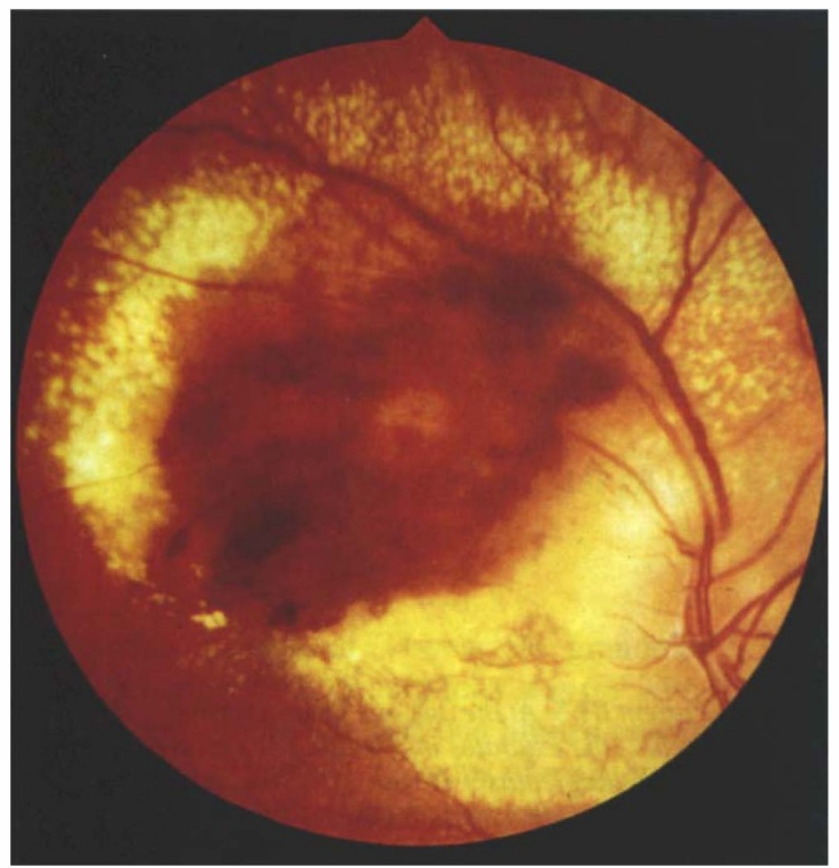

(a)

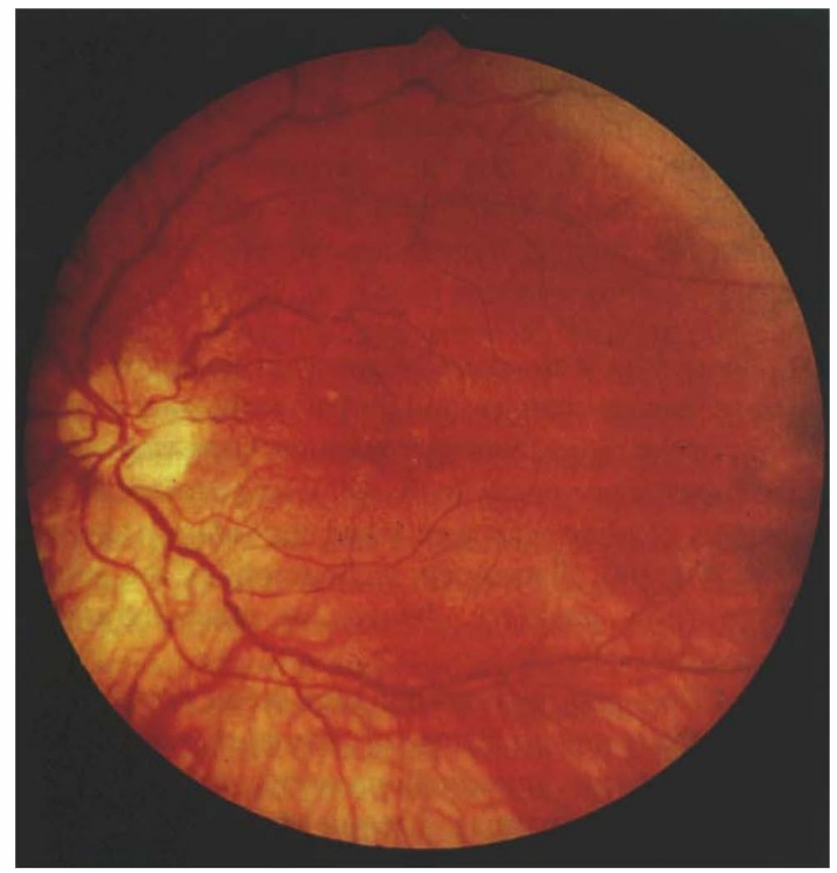

(b)

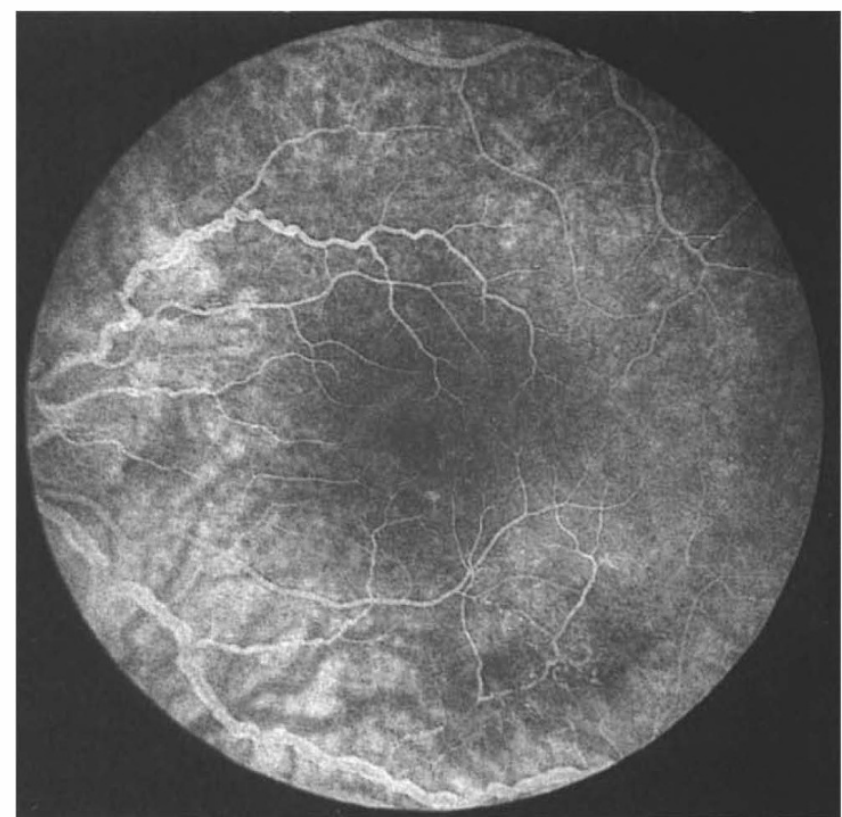

(c)

Fig. 2. Patient III-5, right eye. At the age of 26 years there were exudates and haemorrhage at the upper half of the posterior pole $(a)$. The left eye demonstrated beading of the retinal veins with telangiectasis below and temporal to the fovea $(b, c)$. 


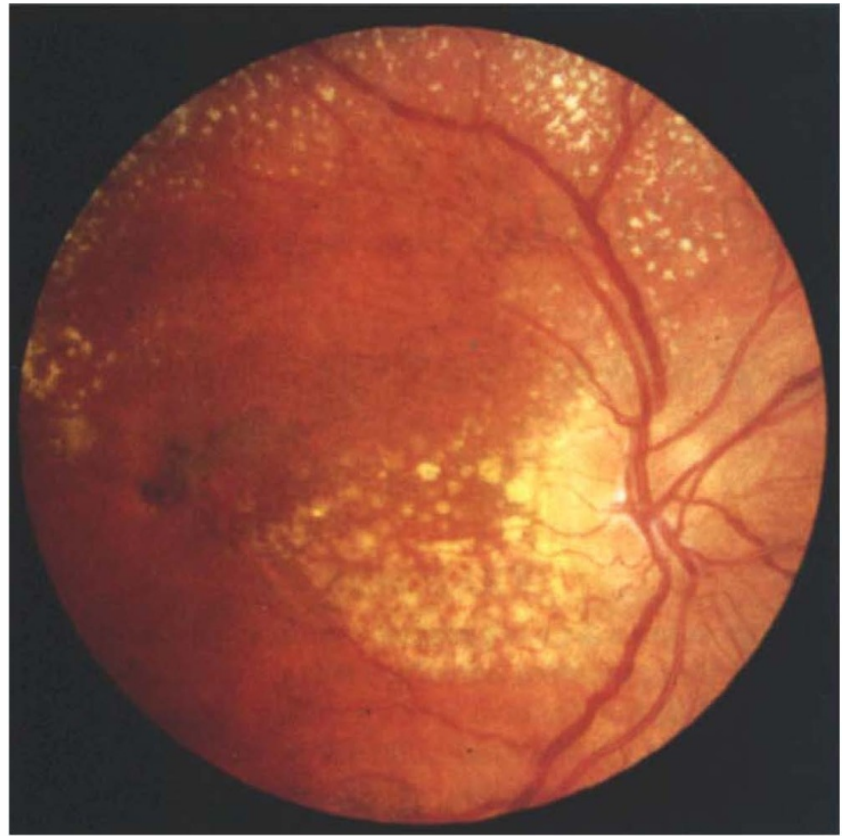

(a)

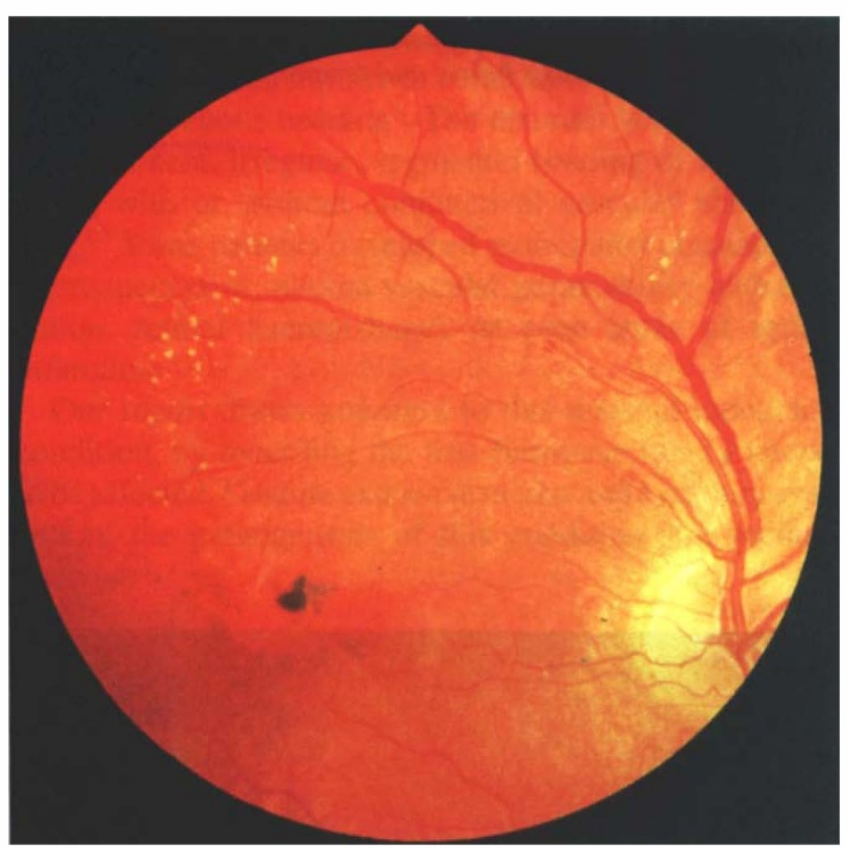

(c)

normal values of $\mathrm{MCV}, \mathrm{MCH}$ and $\mathrm{MCHC}$. Blood pressure was $120 / 75 \mathrm{mmHg}$.

\section{Patient II-2}

The proband's 50-year-old father had lost vision in the right eye 10 years previously, and a diagnosis of retinal venous thrombosis complicated by vitreous haemorrhage and total retinal detachment was made. The right eye had no light perception and left eye visual acuity was $6 / 5$. The bulbar conjunctival vessels showed saccular change in the horizontal meridian bilaterally. The corneas were clear and the intraocular pressures $20 \mathrm{mmHg}$. The right lens was opaque and subluxed posteriorly and the retina was

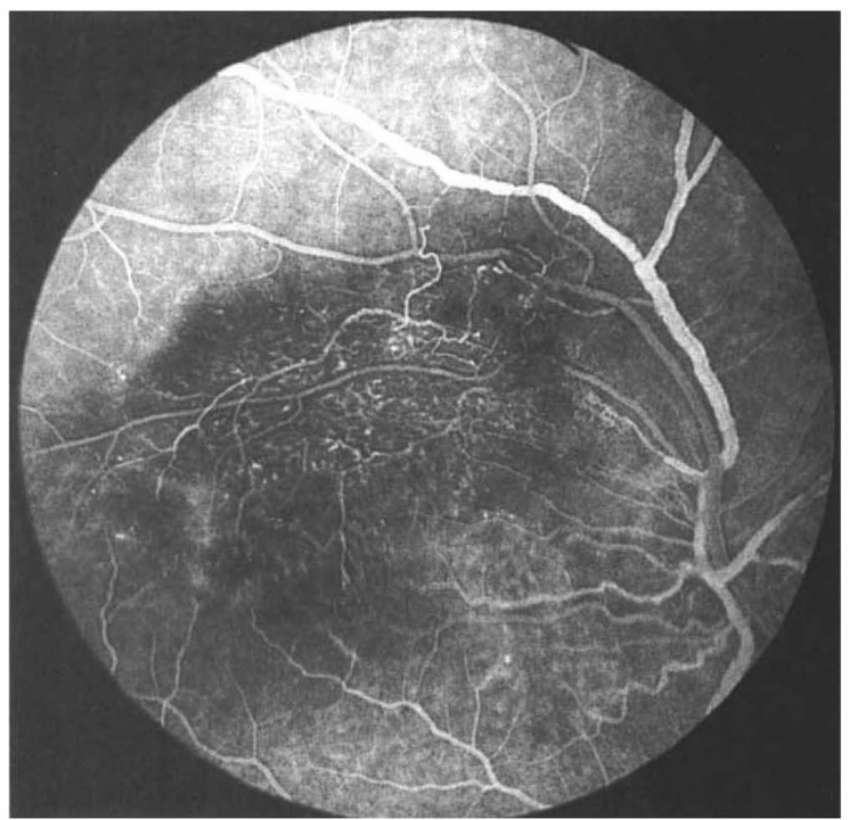

(b)

Fig. 3. Patient III-5. At the age of 27 years right eye vision had improved to 6/9, the exudates had gradually resolved, but there was residual telangiectasis in the affected retina $(a, b)$. Six months later only pigmentary changes were present ( $c$ ).

flat. There was widespread scarring in the right fundus with pigmentary changes, and fibrosis within and on either surface of the retina. Vascular telangiectases were visible in the superior periphery. In the left retina a restricted area of venous beading was seen along the inferior temporal arcade (Fig. 4a). Fluorescein angiography of the left eye did not show capillary changes or other intraretinal abnormalities (Fig. 4b).

Apart from poorly controlled high blood pressure over 10 years there was no history of general disease.

The following investigations were undertaken and showed no abnormalities: haemoglobin and full blood count, clotting studies, plasma electrolytes, lipids and proteins, fasting glucose, $\mathrm{HbA}_{\mathrm{lc}}$, and renal and liver function 


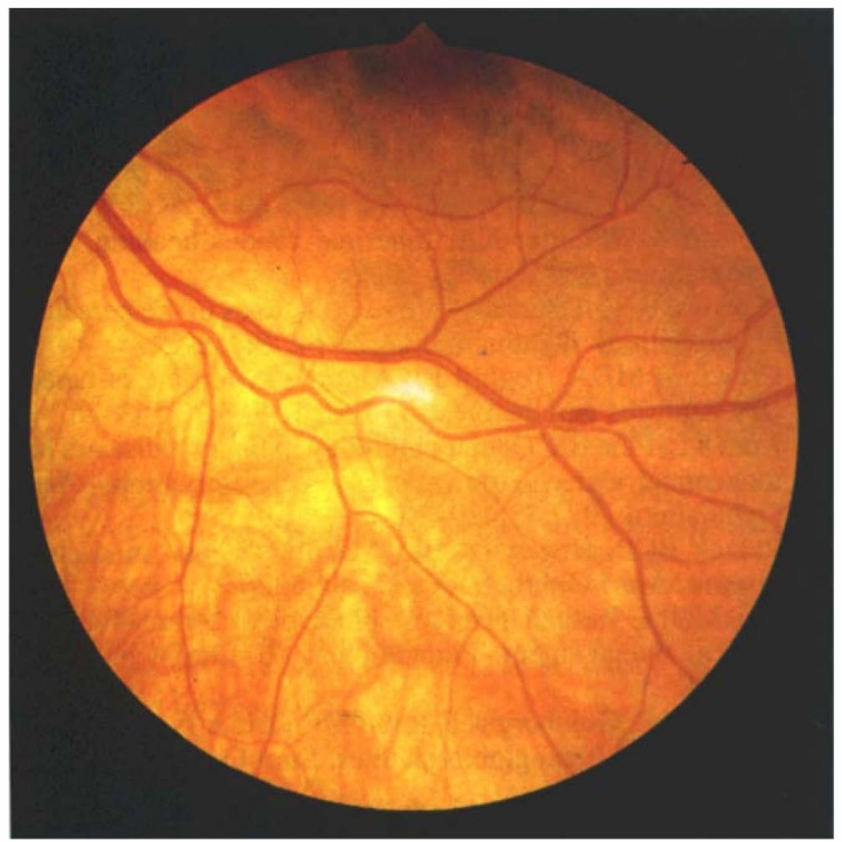

(a)

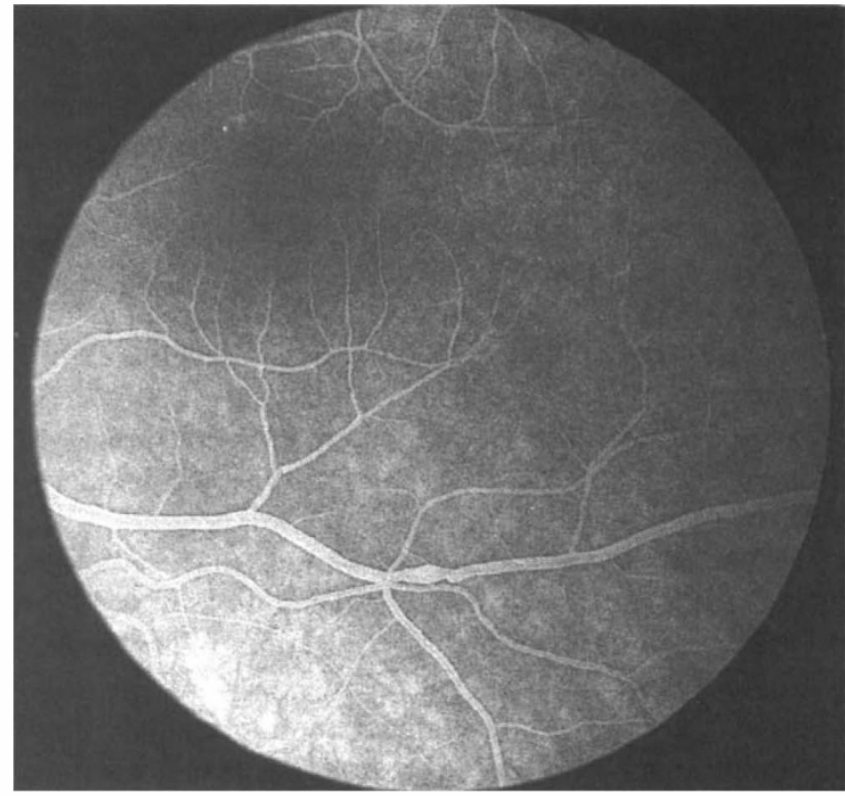

(b)

Fig. 4. Patient II-2. The only vascular abnormality in the posterior pole was one segment of venous beading in the inferior temporal vein $(a, b)$

studies. The patient had proteinuria $>1.0 \mathrm{~g} / 1(>1.4 \mathrm{~g} / 24$ hours), without haematuria.

\section{Patient I-2}

The proband's paternal 74-year-old grandmother was asymptomatic and her visual acuity was $6 / 9$ with the right eye and $6 / 6$ with the left. She had limited beading of one retinal vein in the superior fundus of each eye.

The proband's other known relatives were examined and found to be normal.

\section{DISCUSSION}

Meredith's report ${ }^{1}$ was the first definite description of inherited retinal venous beading and its associated complications. Two members of the reported pedigree had hereditary nephritis, two were deaf and two had low serum neutrophil counts, but none demonstrated all three abnormalities. Meredith considered the possibility that the retinal changes may have been part of a general inherited disorder such as Alport's syndrome which is characterised by renal failure and various ocular abnormalities, ${ }^{3.5}$ although retinal vascular changes have not been described in this condition. ${ }^{6}$ However, the presence of the venous changes in two individuals without kidney disease, and the same degree of severity in males and females (Alport's syndrome is usually less severe in females) led Meredith to consider this association as fortuitous. Some kind of retinal venous tortuosity and beading has been reported in Fabry's disease ${ }^{5-7}$ but vascular complications have never been described, and the disease is inherited in an X-linked fashion. It was concluded that the inherited venous beading probably represented a new disorder with possible systemic abnormalities. Our pedigree, like that described by Stewart and Gitter, ${ }^{2}$ did not demonstrate any associ- ation between retinal venous beading and deafness or renal disease in the proband. The father (case II-2) presented some proteinuria with a reduced creatinine clearance but without haematuria, which was considered to be associated with his hypertension.

Superimposed on the beading or sausage-like configuration of the veins, episodes of apparent acute vascular decompensation have been reported in the previous pedigrees. Those episodes consisted of microaneurysm formation, altered vascular permeability with lipid exudation, focal oedema, areas of retinal infarction, surface retinal neovascularisation and vitreous haemorrhages.

Our proband presented two episodes of this kind over a 9 year period which resolved spontaneously. The nature of the first involving the left eye consisted of intraretinal and subretinal as well as vitreous haemorrhage. The angiogram 9 years later suggested retinal venous obstruction. The second appeared to be the result of retinal branch vein occlusion, complicated by a gross cystoid macular oedema and visual loss. The severity of retinal vascular changes may reflect the pre-existing abnormality of the retinal vessels. The father (case II-2) had also been diagnosed as having retinal vein occlusion, before developing vitreous haemorrhage and retinal detachment.

The risk of severe retinal vascular complications inherent to the syndrome was therefore confirmed in our patients. It has been suggested that low serum neutrophil counts were associated with more prominent venous beading and greater risk of proliferative retinopathy in this condition. This has not been confirmed in our pedigree, in which the counts were normal.

All pedigrees showed marked variability in expression and demonstrate that the dominant nature of the condition may only be shown by surveying the family. Two mem- 
bers of our family showed remarkable spontaneous resolution of disease which in one was associated with visual recovery.

The basic nature of the disorder is not evident. Beading may be produced by segmental narrowing alone or by alternately narrowed and dilated segments. As sympathetic vasomotor innervation is absent in the retinal vessels, changes in calibre must be due either to regulatory responses of the vascular smooth muscle locally, abnormality of circulating blood or possibly to circulating vasoactive agents. It is seen characteristically in diabetes, ${ }^{7}$ venous obstruction, sickle cell retinopathy ${ }^{8}$ or Eales's syndrome. ${ }^{9}$ More rarely it may be related to the hyperviscosity syndrome of the leukaemias and dysproteinaemias, ${ }^{10,11}$ as part of the vascular malformations of some phacomatoses (retinal angiomas in von Hippel-Lindau disease ${ }^{12,13}$ ) or primary retinal telangiectases (Coats' disease $\left.{ }^{14}\right)$.

What still remains poorly understood is whether or not this is the result of a defect in structural elements of the venous walls, leading to an unusual morphological pattern such as beading, or the manifestation of an unknown systemic disease. The results of light and electron microscopic studies of biopsy specimens of conjunctiva and eyelid tissues with abnormal veins ${ }^{1,2}$ showed no evidence of an intrinsic abnormality. On the other hand, no evidence of an associated systemic disorder has been found, despite extensive investigations carried out in the three pedigrees.

B. P. is in receipt of support from the Fonds National Suisse pour la Recherche Scientifique, the Holderbank and the Janggen-
Poehn Stiftung. F.G.H. is in receipt of support from the GertrudKusen-Foundation, Hamburg, Germany, Ho 92/93-01-2.

\section{REFERENCES}

1. Meredith TA. Inherited retinal venous beading. Arch Ophthalmol 1987;105:949-53.

2. Stewart MW, Gitter KA. Inherited venous beading. Am J Ophthalmol 1988;106:675-81.

3. Peterson WS, Albert DM. Fundus changes in the hereditary nephropathies. Ophthalmology 1974;78:762-71.

4. Crawfurd MDA, Toghill PJ. Alport's syndrome of hereditary nephritis and deafness. Q J Med 1968;37:563-76.

5. Ront RL, Fine BS. Ocular pathology in Fabry's disease: histochemical and electron microscopic observations. Am J Ophthalmol 1972;73:419-30.

6. Polack BCP, Hogewind BL. Macular lesions in Alport's disease. Am J Ophthalmol 1977;84:532-5.

7. Tso MOM. Retinal diseases: biomedical foundations and clinical management. Philadelphia: JB Lippincott, 1988: 138-64.

8. Nagpal KC, Goldberg MF, Rabb MF. Ocular manifestations of sickle cell hemoglobinopathies. Surv Ophthalmol 1977; 21:391-411.

9. Elliot AJ. Thirty-year observation of patients with Eales's disease. Am J Ophthalmol 1975;80:404-8.

10. Sanders TE, Podos SM, Rosenbaum LJ. Intraocular manifestations of multiple myeloma. Arch Ophthalmol 1967;77: 789-94.

11. Carr RE, Henkind P. Retinal findings associated with serum hyperviscosity. Am J Ophthalmol 1963;56:23-31.

12. Rosen E. Vascular malformations in the human retina. Am J Ophthalmol 1969;67:501-11.

13. Wing GL, Weiter JJ, Kelly PJ, Albert DM, Gonder GR. Von Hippel-Lindau disease: angiomatosis of the retina and central nervous system. Ophthalmology 1981;88:1311-14.

14. Egerer I, Tasman W, Tormer TL. Coats disease. Arch Ophthalmol 1974;92:109-12. 